\title{
POLINEUROPATIA AMILOIDÓTICA FAMILIAR: UMA REVISÃO DE LITERATURA
}

\author{
Familial amyloidotic polyneuropathy: a literature review
}

\author{
Beatriz Rezende Monteiro'; Olival Cirilo Lucena da Fonseca-Neto²
}

\section{RESUMO}

Introdução: A amiloidose é definida como um grupo heterogêneo de condições de etiologia desconhecida, sendo a polineuropatia amiloidótica familiar uma das formas hereditárias existentes. Essa mutação geralmente leva à polineuropatia sensoriomotora e autonômica progressiva, podendo apresentar casos tanto esporádicos quanto familiares. O espectro clínico é bastante variável, assim como a severidade dos depósitos amilóides. O transplante de fígado e o Tafamidis são as principais opções terapêuticas disponíveis no Brasil. Objetivo: Reforçar os aspectos mais importantes de uma condição tão heterogênea como a PAF, além de revisar também as opções terapêuticas possíveis, de acordo com o estágio da doença. Métodos: Foi revisada literatura no PUBMED, no período de janeiro de 2015 a dezembro de 2019, utilizando a combinação dos descritores Familial amyloid neuropathy AND Liver transplantation AND treatment. Resultados: A PAF é uma condição sistêmica e progressiva, de caráter hereditário, provocada por uma mutação no gene da TTR, sendo Val30Met a variação mais comumente encontrada. O depósito amilóide iniciase muito antes do início do quadro clínico, assim como em outras doenças neurodegenerativas. A sintomatologia da PAF pode variar de acordo com a mutação genética e com os órgãos envolvidos, sendo o coração e os nervos periféricos os principais. O clássico fenótipo dessa condição é caracterizado por neuropatia predominantemente, nas pequenas fibras com perda sensorial dissociada, além de envolvimento autonômico precoce e uma história familiar positiva. No Brasil, o tempo entre o início dos primeiros sintomas e o diagnóstico da PAF é de 5,9 anos, sendo esse tempo um obstáculo para a otimização do manejo da doença. A abordagem do paciente com PAF requer cuidado multidisciplinar com abordagem sintomática, anti-amiloidótica e outras terapias específicas em caso de envolvimento cardíaco, ocular, gastrointestinal e renal. O transplante hepático vem sendo associado a um prognóstico positivo em longo prazo, em pacientes em estágios precoces da doença, jovens, bom status nutricional, curta duração da doença e com a variação Val30Met. Conclusão: Nos dias atuais, apesar de a PAF ser uma doença incurável, é possível atingir o controle da sua progressão, a partir do reconhecimento e diagnóstico precoce associado ao início da terapêutica apropriada. A abordagem multidisciplinar deve sempre ser realizada e as estratégias terapêuticas devem ir além da terapia anti-amiloidótica.

Descritores: Polineuropatia Amiloide Familiar; Transplante de Fígado; Tratamento.

\section{Instituição:}

1- Curso de Medicina do Centro Universitário Maurício de Nassau, Recife/PE, Brasil.

2- Unidade de Transplante de Fígado do Hospital Universitário Oswaldo Cruz, Recife/PE, Brasil.

https://doi.org/10.53855/bjt.v24i4.430

\section{Correspondência:}

Beatriz Rezende Monteiro

E-mail: monteirobeatrizr@gmail.com

\section{INTRODUÇÃO}

A amiloidose é definida como um grupo heterogêneo de condições de etiologia desconhecida e caracterizase pela deposição extracelular e multissistêmica de material proteico, fibrilar, insolúvel, não funcional e resistente à ação de enzimas proteolíticas. ${ }^{1,2}$ Existem formas hereditárias, uma delas a polineuropatia amiloidótica familiar (PAF), e adquiridas da doença, ambas produzindo infiltração amiloide, com consequente disfunção orgânica. Alguns mecanismos podem explicar a propensão dessas proteínas se depositarem, tais como suas propriedades bioquímicas, excessiva síntese protéica e mutações gênicas, como, na transtirretina 
(TTR), a substituição da valina (GTG) pela metionina (ATG) na posição 30 (Val30Met). Essa mutação, geralmente, leva a uma polineuropatia sensoriomotora e autonômica progressiva e pode apresentar casos tanto esporádicos quanto familiares. ${ }^{1,3,4}$

A sintomatologia geralmente inicia-se na terceira ou quarta década de vida, com alta taxa de mortalidade em 10-20 anos do início do quadro. O espectro clínico é bastante variável, assim como a severidade dos depósitos amilóides, gerando, além da polineuropatia sensoriomotora, sintomas gerais como perda de peso, fadiga e úlceras plantares, falência autonômica e cardiomiopatia, a qual é a afecção que representa maior gravidade, ditando um prognóstico mais reservado. ${ }^{1,5-7}$

No Brasil, o Sistema Único de Saúde (SUS) disponibiliza o transplante de fígado e o Tafamidis como as principais opções terapêuticas. No caso do transplante, os resultados dependem do estágio da doença em que o tratamento é proposto, sendo contraindicado nos pacientes com doença severa ou avançada ${ }^{8,9}$ Já o Tafamidis, recentemente aprovado pela Agência Nacional de Vigilância Sanitária (ANVISA), é um potente estabilizador da TTR, sendo a primeira droga a evidenciar redução na progressão da doença, principalmente, em estágios precoces. ${ }^{10}$

\section{OBJETIVO}

Esta revisão busca reforçar os aspectos mais importantes de uma condição tão heterogênea como a PAF, com o objetivo de fornecer suporte aos profissionais de saúde para a suspeita e acurácia diagnóstica, além de revisar também as opções terapêuticas possíveis de acordo com o estágio da doença, buscando melhorar a qualidade de vida dos pacientes através do diagnóstico precoce e tratamento efetivo.

\section{MÉTODO}

Para o presente estudo, foi revisada a literatura no PUBMED, no período de janeiro de 2015 a dezembro de 2019. Foi utilizada a combinação dos descritores Familial amyloid neuropathy AND Liver transplantation AND treatment. Estudos adicionais de relevância para a discussão foram incluídos.

\section{RESULTADOS}

\section{Conceito}

A PAF é uma condição sistêmica e progressiva de caráter hereditário, provocada por uma mutação no gene da TTR, sendo Val30Met a variação mais comumente encontrada, principalmente na Europa e na América Latina. ${ }^{11-15}$ A TTR é uma proteína tetramérica, abundante e solúvel, com a função de transportar a tiroxina, hormônio tireoideano e de ligação ao retinol (complexo da vitamina $A$ ), sendo produzida majoritariamente no fígado $(98 \%)$ e em outros locais alternativos, como o plexo coróide, o epitélio pigmentar da retina e as células $\alpha$ das ilhotas de Langerhans..$^{10,16-19}$ Apesar de originada de uma única proteína, é uma doença de genética heterogênea e de patogênese e manifestações clínicas bastante variáveis. ${ }^{17,20} \mathrm{~A}$ dissociação da TTR mutante em monômeros, a ruptura da barreira hemato-nervosa, o enovelamento proteico incorreto e o fato de ser rica em estruturas folha- $\beta$ possibilitam a agregação e deposição de fibras amiloides insolúveis, com efeitos citotóxicos significantes em diversas estruturas como o fígado, os nervos periféricos, a pele, o trato gastrointestinal, os rins e o miocárdio. ${ }^{13,14,16,20}$

Já foram identificadas aproximadamente 130 mutações nos genes da TTR com fenótipos distintos, variando de acordo com o tipo de mutação e com a idade em que os primeiros sintomas aparecem..$^{13,21,22}$ Nesse sentido, a formação de placas amiloides não está restrita a pacientes senis, podendo ocorrer em qualquer período, sendo mais comum entre a segunda e a nonagésima década de vida. ${ }^{13,17,23}$ O depósito amiloide inicia-se muito antes do início do quadro clínico, assim como em outras doenças neurodegenerativas..$^{13}$ Geralmente, os pacientes que desenvolvem sintomatologia precoce ( $<50$ anos) apresentam neuropatia em pequenas fibras com depósitos amiloides longos e espessos, enquanto os que desenvolvem os sintomas tardiamente ( $>50$ anos) manifestam neuropatia em fibras maiores ou acometimento cardíaco isolado ou ambos, com depósitos amiloides curtos e finos. Além desses fenótipos, outros já definidos são o não-Val30Met misto e o não-Val30Met cardíaco, representado pela mutação Val122lle, principalmente. O reconhecimento desses grupos fenotípicos é de extrema importância, pois permite a identificação dos exames mais relevantes para o acompanhamento a longo prazo dos pacientes, de acordo com seu fenótipo. De toda forma, com a progressão da doença, a tendência é de que os fenótipos isolados se tornem cada vez mais mistos, de modo que, no estágio I os pacientes ainda possam ser acompanhados ambulatorialmente, ao contrário do estágio III, no qual estão acamados e com comprometimento multissistêmico..$^{13,23}$

Epidemiologicamente, a PAF é nos dias atuais, uma enfermidade global, reconhecida em todos os continentes. ${ }^{15}$ Sua prevalência estimada é de 0.9-1.1 a cada 10.000 .000 de pessoas, com sobrevivência 
estimada de 7-12 anos após o início dos sintomas. No entanto, esses dados são subestimados, devido ao fato de que muitos pacientes só recebem o diagnóstico após a realização da autópsia, o que torna a PAF uma doença subdiagnosticada. ${ }^{14,17}$ Porém, é válido ressaltar que esse cenário vem mudando, a partir do maior conhecimento acerca da PAF e da ampliação de técnicas não invasivas para a avaliação e diagnóstico desses pacientes. ${ }^{16}$ Existem países que possuem epidemiologia maior para PAF do que outros, como mostrado na Figura 1.

Figura 1: Países no mundo com maior epidemiologia para PAF.

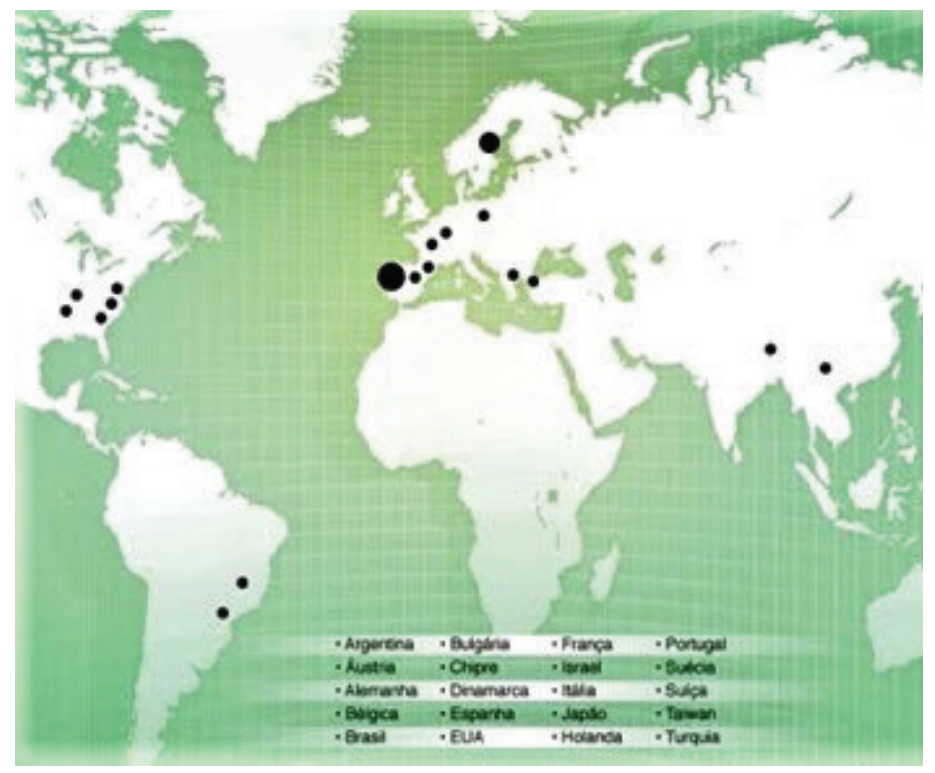

Fonte: Associação Portuguesa de Paramiloidose. Disponível em: $<$ http://www.paramiloidose.com/dia_nacional.php? $a=8 \& a d m=1>$.

\section{Quadro clínico}

O quadro clínico da PAF pode variar de acordo com a mutação genética e com os órgãos envolvidos, sendo o coração e os nervos periféricos os principais. ${ }^{24,25}$ Os sintomas iniciais comumente são dor e parestesia nos pés, associados a perda sensorial térmica e, em seguida, leve perda da sensibilidade tátil e hiporreflexia ou arreflexia do tornozelo, além de perda de peso, diarreia, constipação, sensação de impotência, hipotensão ortostática e boca e olhos secos. ${ }^{10} \mathrm{Em}$ alguns casos, sintomas gastrointestinais podem estar presentes antes mesmo do aparecimento da polineuropatia periférica, tais como diarreia prolongada, incontinência fecal e má nutrição grave. Em outros, as manifestações autonômicas também podem representar o início do quadro clínico da PAF, como por exemplo, a falha da função sudomotora. ${ }^{25,26}$ A figura 2 mostra os principais sintomas e órgãos e sistemas envolvidos.
Figura 2: Principais sinais e sintomas em órgãos e sistemas acometidos

\begin{tabular}{|c|}
\hline $\begin{array}{c}\text { Sistema Nervoso } \\
\text { Periférico }\end{array}$ \\
\hline $\begin{array}{c}\text {-Neuropatia periférica } \\
\text { sensoriomotora } \\
\text {-Síndrome do Túnel } \\
\text { do Carpo }\end{array}$ \\
\hline \\
\hline $\begin{array}{c}\text { Sistema nervoso } \\
\text { autônomo }\end{array}$ \\
-Hipotensão \\
ortostática \\
-Disfunção erétil e \\
sexual \\
-Anormalidades \\
sudomotoras
\end{tabular}
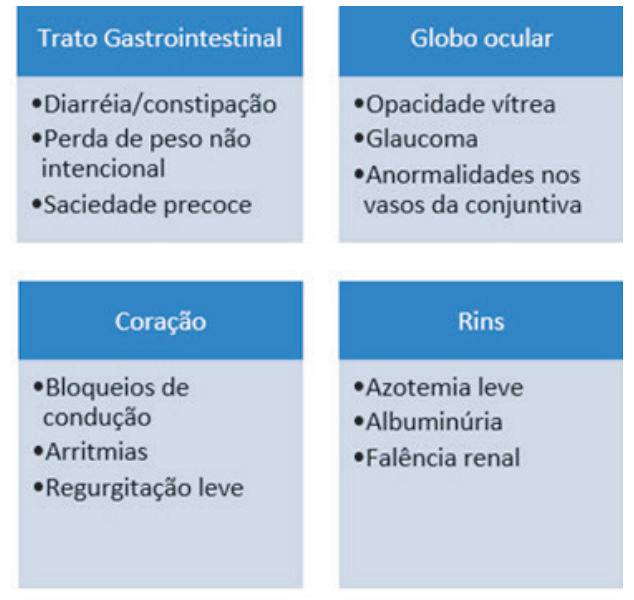
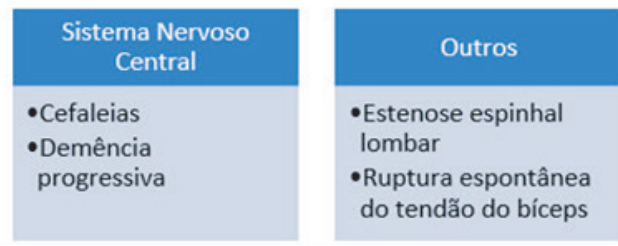

PAF: Polineuropatia amiloidótica familiar.

Fonte: Elaborada pelo autor.

O clássico fenótipo da amiloidose portuguesa, principalmente no Brasil, em Portugal e no Japão em pacientes com desenvolvimento precoce dos sintomas, é caracterizado por neuropatia, predominantemente nas pequenas fibras, com perda sensorial dissociada, além de envolvimento autonômico precoce e história familiar positiva. Em pacientes com desenvolvimento tardio, grave perda sensorial, leves manifestações autonômicas, envolvimento cardíaco severo e ausência de história familiar são evidenciados com mais frequência. ${ }^{10} \dot{E}$ importante ressaltar que uma mesma mutação genética pode apresentar diversos fenótipos distintos entre si, inclusive dentro da mesma família. ${ }^{23}$ A clássica polineuropatia da amiloidose portuguesa é simétrica em membros inferiores, inicialmente com progressão para os superiores em 4-5 anos, sendo axonal, ou seja, comprometendo todas as funções pertencentes à fibra nervosa (motora, sensorial e autonômica). ${ }^{27,28}$ Há cinco indicadores de progressão da doença: perda de peso contínua e não intencional; aparecimento de novos ou piora dos sintomas autonômicos, tais como hipotensão ortostática, diarreia e impotência; progressão da neuropatia simpática sensoriomotora; desenvolvimento de complicações oftalmológicas e aparecimento ou evolução de manifestações cardíacas. ${ }^{23}$

A progressão da doença pode ser avaliada através de alguns testes e escores como o escore de comprometimento neuropático (ECN), escore 
de sintomas neuropáticos, escore de deficiência neurológica entre outros, cada um com suas respectivas vantagens na avaliação prognóstica. ${ }^{23}$ Além disso, existem fatores que podem auxiliar na diferenciação entre a PAF e outros diagnósticos diferenciais, tais como a ausência de ataxia, dor neuropática (muitas vezes descrita como dor relâmpago), perda sensorial das fibras menores acima do punho, fraqueza nos membros superiores e, o já citado comprometimento autonômico, prejudicando o trato gastrointestinal e a função erétil, o que afeta significativamente a qualidade de vida do paciente. ${ }^{18}$ Dessa forma, apesar dos principais sintomas serem associados à neuropatia, os profissionais de saúde devem estar atentos a manifestações cardíacas, oftalmológicas e renais, já que a PAF é uma condição multissistêmica, e portanto, necessita de uma abordagem multidisciplinar.

O envolvimento cardíaco, na maioria das vezes, permanece latente por longo período, sendo subdiagnosticado, porém presente em quase $50 \%$ dos casos, devendo ser avaliado o quanto antes através de biomarcadores e exames de imagem. Quando os sintomas aparecem, geralmente já se tem insuficiência cardíaca com fração de ejeção preservada e/ou cardiomiopatia hipertrófica, síncope cardiogênica e edema periférico instalados. ${ }^{13}$ Indivíduos com PAF têm alta incidência de morte por causas cardiovasculares (38\%), sendo, portanto, a neuropatia autonômica cardiovascular a mais ameaçadora manifestação autonômica, com risco de morte súbita. ${ }^{20,25} \mathrm{~A}$ hipotensão ortostática, por exemplo, é uma importante consequência da hipoperfusão cardíaca, provocando tonturas, visão embaçada em ortostase, queda e síncope, podendo ser agravada por episódios diarreicos ou por tratamento farmacológico concomitante para outras condições, como a hipertensão arterial sistêmica flutuante. A presença de anormalidades cardíacas como os distúrbios de condução, disautonomia, ruptura do tendão distal do bíceps e síndrome do túnel do carpo ou síndrome do túnel do carpo bilateral associada à neuropatia sensoriomotora simétrica e progressiva e/ou cardiomiopatia deve sugerir o diagnóstico de PAF.16-18

A amiloidose cardíaca é subdividida em dois tipos: amiloidose sistêmica senil (TTR não mutante ou wild type) e a TTR mutante ou hereditária. A primeira, clinicamente identificada por síndrome do túnel do carpo bilateral, ruptura do tendão do bíceps e estenose espinhal, tem início da sintomatologia por volta da sétima década de vida. Além disso, a amiloidose não mutante cursa com falência cardíaca congestiva, fibrilação atrial e arritmia intratável. Já a mutante, geralmente envolve o sistema nervoso autônomo e periférico, dependendo, principalmente, da mutação envolvida. ${ }^{29} \mathrm{Em}$ ambos subtipos, as manifestações cardíacas são bastante predominantes. ${ }^{17}$ No Brasil, as anormalidades cardíacas mais comuns são as repolarizações ventriculares não específicas, distúrbios de condução ventricular, taquicardia atrial, complicações valvares e aumento da ecogenicidade miocárdica. ${ }^{10} \mathrm{~A}$ PAF é uma condição facilmente confundida com outras causas de cardiomiopatia infiltrativa e hipertrófica e por isso, a coexistência de outros sintomas sistêmicos associados ao sistema nervoso periférico e autonômico, concomitantes à disfunção cardíaca é de grande valia para o raciocínio clínico e diagnóstico. ${ }^{27}$

A síndrome do túnel do carpo, neuropatia do nervo mediano é um achado comum e precoce nos pacientes, podendo preceder as complicações cardíacas em seis anos na amiloidose cardíaca não mutante. ${ }^{17}$

As manifestações oculares na PAF incluem principalmente síndrome do olho seco (70\%), glaucoma (20\%) e amiloidose vítrea (17\%), devido a anormalidades na retina e na microvasculatura coroidal. ${ }^{13,29}$

A sintomatologia autonômica gastrointestinal é bastante frequente e variada, podendo, inicialmente, ser identificada como gastroparesia, através de sintomas como saciedade precoce e lentidão na digestão. Esse quadro geralmente progride para náusea pós-prandial, com vômitos recorrentes, desidratação e anorexia, chegando a estágios avançados com perda severa de peso não intencional, má nutrição e deficiência vitamínica, causando grande impacto na sobrevida do paciente. O hábito intestinal pode também se alterar, tanto por um quadro diarreico explosivo e persistente quanto por constipação. ${ }^{25}$ Uma forma interessante de avaliar a progressão da PAF é através do Índice de Massa Corporal (IMC), sendo o IMC modificado (quando há a multiplicação pela albumina sérica, a fim de compensar o edema), fortemente, correlacionado à sobrevivência do paciente e com o desfecho póstransplante hepático. ${ }^{23,30}$

A monitoração da função renal é fundamental, já que a proteinúria e o comprometimento renal são características clínicas importantes na PAF, podendo a microalbuminúria preceder a neuropatia. ${ }^{23}$ Apesar de rara em pacientes com início tardio dos sintomas, a síndrome nefrítica e a falência renal progressiva são também possibilidades. ${ }^{29}$

O quadro clínico da PAF não é único, porém, em pacientes que apresentam polineuropatia sensoriomotora progressiva e indeterminada, além de um ou mais dos sinais de alerta (Tabela 1), deve-se suspeitar de PAF e considerar biópsia e testes genéticos. ${ }^{10}$ 
Tabela 1: Suspeição da PAF e sinais de alerta.

\section{Suspeição de Polineuropatia Amiloidótica Familiar (PAF)}

Além de um ou mais dos sinais de alerta abaixo:

História familiar de polineuropatia

Hipotensão ortostática

Disfunção sexual ou erétil

\begin{tabular}{|c|c|}
\hline Polineuropatia & Perda ponderal inexplicada \\
\hline sensoriomotora & $\begin{array}{l}\text { Arritmias, bloqueios de condução, } \\
\text { hipertrofia cardíaca ou miocardiopatia }\end{array}$ \\
\hline progressive & Síndrome do túnel do carpo bilateral \\
\hline terminada & $\begin{array}{l}\text { Anormalidades renais (proteinúria ou } \\
\text { azotemia) }\end{array}$ \\
\hline & Opacidade vítrea \\
\hline & Queixas gastrointestinais \\
\hline & Progressão rápida \\
\hline & Falha do tratamento de primeira linha \\
\hline
\end{tabular}

Fonte: elaborado pelo autor.

\section{Diagnóstico}

Em áreas endêmicas, o diagnóstico de PAF é feito em um ano a partir do início dos sintomas, e geralmente nesses países há monitoramento regular dos familiares de pacientes já diagnosticados em centros de referência. Já em áreas não endêmicas, o diagnóstico em relação às endêmicas pode atrasar 3-4 anos. ${ }^{13}$ No Brasil, o tempo entre o início dos primeiros sintomas e o diagnóstico da PAF é de 5,9 anos, o que é um enorme obstáculo para a otimização do manejo da doença..$^{10,12}$ Isso acontece pela ausência de história familiar, pela variedade de apresentações clínicas que podem mimetizar outras neuropatias periféricas, diagnóstico errado, dentre outros motivos. ${ }^{13}$

Tabela 2: Investigação e confirmação diagnóstica.

Investigação diagnóstica por exames complementares

\section{Biópsia}

Testes patológicos

Coloração Vermelho do Congo

Imuno-histoquímica com anticorpo anti-TTR

Testes genéticos

PCR-RFLP

PCR em tempo real

Sequenciamento

PCR-SSP

TTR: transtiterrina; PCR: Reação em cadeia de polimerase; RFLP: Polimorfismo de comprimento de fragmento de limitação (técnica); SSP: Iniciadores específicos da sequência. Fonte: Elaborada pelo autor.
O diagnóstico da PAF é realizado em duas etapas. A primeira é através da suspeição clínica, como mostrado na Tabela 1 e a segunda através dos exames diagnósticos (Tabela 2), como a biópsia, exame de alta acurácia em sítios onde houve o depósito amiloide, sendo a glândula salival menor, os nervos, o trato gastrointestinal e a gordura abdominal os mais frequentemente biopsiados, além do sequenciamento do gene TTR para a identificação de mutações amilodoigênicas. Apesar disso, uma biópsia negativa não exclui PAF, pois a sensibilidade do exame varia de acordo com o tecido biopsiado, a mutação envolvida, a idade do paciente e a experiência do médico patologista. Dessa forma, múltiplas biópsias podem ser necessárias. ${ }^{12,13}$ Há ainda o estudo patológico através de técnicas como a de imunohistoquímica com anticorpos anti-TTR e a coloração Vermelho do Congo nas peças biopsiadas. ${ }^{12}$

Medidas e testes adicionais, como os biomarcadores também podem ser utilizados a fim de avaliar a severidade e a progressão da patologia e dos sintomas. ${ }^{13}$ Um exemplo desses biomarcadores seria a expressão da proteína protocadherina-10, que tem sido associada à progressão da neuropatia periférica na PAF. ${ }^{17}$

A avaliação cardíaca deve incluir o exame clínico, a partir da classificação da New York Heart Association (NYHA), eletrocardiograma (ECG), ecocardiograma (ECO), teste de caminhada de seis minutos (TC6M) e testes laboratoriais, como o fragmento $\mathrm{N}$-terminal do peptídeo natriurético tipo B (NT-ProBNP). ${ }^{23}$

Em muitos casos, a biópsia negativa contribui para que o paciente seja diagnosticado com diversas condições, que são em muitos aspectos similares à PAF, como por exemplo a Polineuropatia Inflamatória Desmielinizante Crônica (CIDP). ${ }^{13,28,31}$ Segundo Plante-bordeneuve et al, até $37 \%$ dos casos de PAF preenchem todos os critérios necessários para CIDP, devido ao comprometimento sensório-motor com arreflexia. No entanto, é válido ressaltar que esses pacientes continuarão evoluindo com o déficit neurológico, mesmo diante do uso de imunoglobulinas e corticoides. ${ }^{26}$

Os testes genéticos são necessários para diferenciar a amiloidose mutante da não mutante, o que pode prever o curso clínico da doença em cada paciente. ${ }^{21}$

Após o diagnóstico, é necessário determinar o estágio da neuropatia e a extensão sistêmica da doença, o que servirá de guia para o tratamento mais recomendado. ${ }^{12}$

\section{Tratamento}

O manejo da PAF requer cuidado multidisciplinar com abordagem sintomática, anti-amiloidótica e 
outras terapias específicas, em caso de envolvimento cardíaco, ocular, gastrointestinal e renal (Tabela 3 ). $\mathrm{O}$ aconselhamento genético do paciente e dos seus parentes é altamente recomendado. Além disso, é indispensável a orientação e educação do paciente em relação a essa condição..$^{12,13}$

Tabela 3: Opções terapêuticas da PAF.

\begin{tabular}{lll}
\hline Terapia sintomática & $\begin{array}{l}\text { Terapia } \\
\text { antiamiloidótica }\end{array}$ & $\begin{array}{l}\text { Manejo dos órgãos } \\
\text { envolvidos }\end{array}$ \\
\hline Manejo da dor & Transplante hepático & Marcapasso \\
$\begin{array}{l}\text { Reabilitação } \\
\begin{array}{l}\text { Tratamento específico } \\
\text { para as disfunções } \\
\text { autonômicas }\end{array}\end{array}$ & Diflunisal & Diálise \\
& Patisiran & Transplante renal \\
& Inotersen & Transplante cardíaco \\
\hline
\end{tabular}

Diversas terapias modificadoras da doença têm sido desenvolvidas ao logo dos últimos anos e a primeira delas foi o transplante hepático em $1990 .^{10,20}$ O fígado de um paciente com PAF do ponto de vista morfológico e funcional é excelente e pode ser doado para pacientes selecionados, desde que haja cuidadoso esclarecimento para o receptor e seu respectivo consentimento. A fim de compensar o cenário da falta de doadores hepáticos, propõe-se que os fígados com PAF sejam transplantados em pacientes com enfermidades hepáticas graves, em lista de espera e limitada expectativa de vida, definindo o processo conhecido como transplante hepático em dominó. ${ }^{8,32}$ Esse procedimento é responsável por remover a principal fonte de TTR mutada, eliminar $90 \%$ da circulante no sangue e prolongar a sobrevida em 20 anos (55\%), além de evidenciar melhora na neuropatia sensorial e motora, sendo, portanto, a terapia padrão para PAF. ${ }^{14,16,21}$

O transplante hepático vem sendo associado a um prognóstico positivo a longo prazo, em pacientes em estágios precoces da doença, jovens $(<50$ anos de idade), com bom status nutricional, curta duração da doença e com a variação Val30Met. ${ }^{16,17,26}$ Entretanto, há a possibilidade de a deposição de placas amiloides nos tecidos prosseguir após o transplante de fígado pela promoção de TTR wild type, e nesses casos, o transplante já não é mais uma opção, principalmente, em pacientes de início sintomático tardio. ${ }^{19}$

Em pacientes com cardiomiopatia severa provocada pela PAF, o transplante ortotópico cardíaco associado ao transplante de fígado pode ser recomendado, à exceção de casos com a mutação V122I ou TTR wild type, já que esses pacientes tendem a não responder a essa terapêutica. ${ }^{29}$

Por outro lado, Vollmar et al analisaram em um período de cinco anos pré e pós-transplante hepático pacientes que possuíam a variação Val30Met e outros que não a possuíam, concluindo que não houve diferença significativa nos sintomas avaliados, o que sugere estabilização da doença pós-transplante. Ademais, Barnejee et al evidenciaram que indivíduos submetidos a dois transplantes (hepático e cardíaco ou hepático e renal) simultaneamente, quando indicado, possuem melhor sobrevida quando comparados a pacientes que realizam transplante de fígado isolado. ${ }^{33}$

Apesar de efetivo, o transplante hepático possui diversas limitações, como não reverter os danos já causados nos órgãos, a escassez de doadores, os efeitos adversos das medicações imunossupressoras, o custo do transplante e de outras terapêuticas adicionais e a dificuldade de identificar os pacientes candidatos na fase inicial da doença. ${ }^{34}$ Além disso, é um procedimento complexo, que envolve uma taxa de mortalidade de $16 \%$ em um ano e de $27 \%$ em cinco anos, sendo os eventos cardíacos a principal causa $(38 \%) .{ }^{20}$

Já que nem todos os pacientes são elegíveis para procedimentos invasivos, novas modalidades terapêuticas vêm sendo exploradas. Uma delas é a estabilização do tetrâmero da proteína TTR a partir de pequenas moléculas que agem como estabilizador cinético e impedem a dissociação em monômeros com incorreto enovelamento. ${ }^{13,34}$ Diflunisal é um antiinflamatório não esteroidal, não seletivo, descoberto em 1970 e utilizado off label nos estágios I e II da doença, evitando a formação de novas placas amiloides. No entanto, seu uso a longo prazo é associado a aumento da ocorrência de efeitos indesejados gastrointestinais, renais e cardiotóxicos, com alta taxa (57\%) de descontinuação. ${ }^{10,12,18,21,34}$ Outro estabilizador da TTR, porém sem os efeitos adversos presentes no Diflunisal, é o Tafamidis (estabilizador seletivo), usado em pacientes em estágio $1 \mathrm{com}$ polineuropatia sintomática. ${ }^{10} \mathrm{~A}$ terapia com Tafamidis, aprovada em países como Brasil, Japão, Argentina, México e no continente Europeu promove redução da deterioração neurológica, preservação da função do nervo, manutenção da qualidade de vida de pacientes em estágios iniciais com a variação Val30Met, estabilizando a TTR em $98 \%$ dos casos. ${ }^{18,21,35}$ Além disso, o Tafamidis também mostrou minimizar todas as causas de mortalidade e hospitalizações por complicações cardiovasculares. ${ }^{34}$ 
Outra alternativa terapêutica é o silenciamento gênico nos estágios I e II da PAF, suprimindo a produção de TTR mutante e não-mutante pelos hepatócitos. ${ }^{18,19,26}$ O Patisiran contém uma sequência de RNA alvo encapsulado em nanopartícula lipídica, sendo administrado por infusão intravenosa três vezes por semana; tem sido associado a reduções significativas na concentração sérica da TTR sem eventos adversos importantes, além de provocar paralisação ou regressão do quadro evolutivo da PAF.13,34 Já o Inortesen é outra droga utilizada no silenciamento gênico administrada por via subcutânea uma vez por semana, agindo como inibidor do oligonucleotídeo antisense, reduzindo o curso da doença neurológica e melhorando a qualidade de vida dos pacientes com PAF.10,18 Ambas as drogas, Patisiran e Inortesen, têm demonstrado eficácia em pacientes em estágio de comprometimento neurológico precoce e tardio da PAF. ${ }^{18}$

Várias outras terapias para o tratamento da PAF estão com estudos em andamento, tais como anticorpos monoclonais, AG-10 (novo estabilizador da TTR), epigalocatequina-3-galato (outro estabilizador da TTR), CHF5074 (modulador da gama-secretase), entre outros. ${ }^{16,18,21}$

\section{CONCLUSÃO}

Esta revisão reforçou a compreensão acerca da PAF desde o seu conceito atéa patogênese, a heterogeneidade dos seus sinais e sintomas, as principais ferramentas diagnósticas, assim como as opções terapêuticas disponíveis e em desenvolvimento. Muitos avanços têm sido realizados nos últimos anos, o que tem permitido melhor entendimento da formação e deposição das fibras amiloides nesses casos, facilitando o diagnóstico e a elaboração de novas abordagens terapêuticas menos invasivas que o transplante hepático.

Atualmente, a PAF pode ter a sua progressão controlada a partir do reconhecimento e diagnóstico precoce associado ao início da terapêutica apropriada, mesmo em pacientes com início tardio dos sintomas, antes que danos irreversíveis se desenvolvam. Entretanto, muitos pacientes com PAF ainda são esquecidos ou mal diagnosticados com outras enfermidades, o que potencializa a importância deste artigo.

A abordagem multidisciplinar deve sempre ser realizada e as estratégias terapêuticas devem ir além da terapia anti-amiloidótica, incluindo o tratamento sintomático e o manejo de possíveis complicações.

\section{ABSTRACT}

Introduction: Amyloidosis is defined as a heterogeneous group of conditions of unknown etiology, and familial amyloidotic polyneuropathy is one of the existing hereditary forms. This mutation usually leads to a progressive sensorimotor and autonomic polyneuropathy, which can present both sporadic and familial cases. Clinical spectrum is quite variable, as it is the severity of amyloid deposits. Liver transplantation and Tafamidis are the major therapeutic options available in Brazil. Purpose: To reinforce the most important aspects of a condition as heterogeneous as FAP further to also reviewing the possible therapeutic options according to the stage of the disease. Methods: The literature on PUBMED from January 2015 to December 2019 was reviewed by using the combination of descriptors Familial amyloid neuropathy AND Liver transplantation AND Treatment. RESULTS: FAP is a systemic and progressive condition of hereditary nature, caused by a mutation in the TTR gene with Val30Met being the most commonly found variation. Amyloid deposits start long before the onset of the clinical picture, as in other neurodegenerative diseases. FAP symptoms can vary according to the genetic mutation and the organs involved, with the heart and peripheral nerves being the main ones. The classic heel disease phenotype is characterized by neuropathy, predominantly in small fibers with dissociated sensory loss, added to early autonomic involvement and a positive family history. In Brazil, the time between the onset of the first symptoms and the FAP diagnosis is 5.9 years, which is an obstacle to optimizing the management of the disease. The approach of FAP requires a multidisciplinary care with a symptomatic approach, anti-amyloidosis and other specific therapies in case of cardiac, ocular, gastrointestinal and renal involvement. Liver transplantation has been associated to a positive long-term prognosis in patients in early disease stages, young people, good nutritional status, short disease duration, and with the Val30Met variation. Conclusion: Nowadays, despite the fact that FAP is an incurable disease, it is possible to control its progression through early recognition and diagnosis associated to the initiation of an appropriate therapy. A multidisciplinary approach must always be carried out and therapeutic strategies must go beyond anti-amyloidotic therapy.

Keywords: Amyloid Neuropathies, Familial; Liver transplantation; Treatment. 


\section{REFERÊNCIAS}

1. Quinto AAM, Municio AM, Romero LCJ, Segurola CL, Pulido JC, Alonso IJ, et al. Trasplante hepático como tratamiento de la polineuropatía amiloidótica familiar en pacientes mayores de 60 años. Med Clin (Barc). 2015;144(9):385-8.

2. Coutinho MCA, Dias NC, Cantinho G, Conceição I, Guimarães T, Lima da Silva G, et al. Progression of myocardial sympathetic denervation assessed by 123I-MIBG imaging in familial amyloid polyneuropathy and the effect of liver transplantation. Rev Port Cardiol. 2017;36(5):333-40.

3. Adams D, Beaudonnet G, Adam C, Lacroix C, Théaudin M, Cauquil C, et al. Familial amyloid polyneuropathy: When does it stop to be asymptomatic and need a treatment? Rev Neurol (Paris). 2016;172(10):645-52.

4. Santos MO, Brito D. Severe heart disease in an unusual case of familial amyloid polyneuropathy type I. Rev Port Cardiol. 2013;32(9):729-33.

5. Rodríguez DLH, Domínguez RG, Miras PM, Sosa LG. Manejo perioperatorio del trasplante hepático en la polineuropatía amiloidótica familiar con afectación cardíaca. Rev Esp Anestesiol Reanim. 2015;62(1):46-8.

6. García-Pavía P, Beamud FM, Casasnovas C, representació del Grupo de Estudio Tratamiento de la Polineuropatía Amiloidótica Familiar por Transtiretina en. Recomendaciones para el diagnóstico y tratamiento de la polineuropatía amiloidótica familiar por transtiretina Recommendations regarding diagnosis and treatment of transthyretin familial amyloid polyneuropathy. Med Clin (Barc). 2015;145(5):211-7.

7. Finsterer J, Iglseder S, Wanschitz J, Topakian R, Löscher WN, Grisold W. Hereditary transthyretin-related amyloidosis. Acta Neurol Scand. 2019;139(2):92-105.

8. Chen Q, Yuan L, Deng X, Yang Z, Zhang S, Deng S, et al. A Missense Variant p.Ala117Ser in the Transthyretin Gene of a Han Chinese Family with Familial Amyloid Polyneuropathy. Mol Neurobiol. 2018;55(6):4911-7.

9. Adams D, Théaudin M, Cauquil C, Algalarrondo V, Slama M. FAP neuropathy and emerging treatments. Curr Neurol Neurosci Rep. 2014;14(3).

10. Pinto MV, Barreira AA, Bulle AS, de Freitas MRG, França MC, Gondim F de AA, et al. Brazilian consensus for diagnosis, management and treatment of transthyretin familial amyloid polyneuropathy. Arq Neuropsiquiatr. 2018;76(9):609-21.

11. Escolano-Lozano F, Barreiros AP, Birklein F, Geber C. Transthyretin familial amyloid polyneuropathy (TTR-FAP): Parameters for early diagnosis. Brain Behav. 2018;8(1):1-9.

12. Adams D, Suhr OB, Hund E, Obici L, Tournev I, Campistol $\mathrm{JM}$, et al. First european consensus for diagnosis, management, and treatment of transthyretin familial amyloid polyneuropathy. Curr Opin Neurol. 2016 Feb 1;29:S14-26.

13. Adams D, Koike H, Slama M, Coelho T. Hereditary transthyretin amyloidosis: a model of medical progress for a fatal disease. Nat Rev Neurol. 2019;15(7):387-404.
14. Mnatsakanova D, Živković SA. latrogenic amyloid polyneuropathy after domino liver transplantation. World J Hepatol. 2017;9(3):126-30.

15. Plante-Bordeneuve V. Transthyretin familial amyloid polyneuropathy: an update. J Neurol. 2018;265(4):976-83.

16. Emdin M, Aimo A, Rapezzi C, Fontana M, Perfetto F, Seferović PM, et al. Treatment of cardiac transthyretin amyloidosis: An update. Eur Heart J. 2019;40(45):3699_ 706.

17. Galant NJ, Westermark P, Higaki JN, Chakrabartty A. Transthyretin amyloidosis: An under-recognized neuropathy and cardiomyopathy. Clin Sci. 2017;131(5):395-409.

18. Gertz MA, Scheinberg M, Waddington-Cruz M, Heitner SB, Karam C, Drachman B, et al. Inotersen for the treatment of adults with polyneuropathy caused by hereditary transthyretin-mediated amyloidosis. Expert Rev Clin Pharmacol. 2019;12(8):701-11.

19. Koike $\mathrm{H}$, Katsuno M. Ultrastructure in transthyretin amyloidosis: From pathophysiology to therapeutic insights. Biomedicines. 2019;7(1).

20. Algalarrondo V, Antonini T, Théaudin M, Chemla $D$, Benmalek A, Castaing D, et al. Cause of death analysis and temporal trends in survival after liver transplantation for transthyretin familial amyloid polyneuropathy. Amyloid. 2018;25(4):253-60.

21. Gertz MA, Mauermann ML, Grogan M, Coelho T. Advances in the treatment of hereditary transthyretin amyloidosis: A review. Brain Behav. 2019;9(9):1-12.

22. Banerjee D, Roeker LE, Grogan M, Swiecicki P, Poterucha $\mathrm{J}$, Heimbach J, et al. Outcomes of patients with familial transthyretin amyloidosis after liver transplantation. Prog Transplant. 2017;27(3):246-50.

23. Conceição I, Coelho T, Rapezzi C, Parman Y, Obici L, Galán L, et al. Assessment of patients with hereditary transthyretin amyloidosis-understanding the impact of management and disease progression. Amyloid. 2019;26(3):103-11.

24. Nakov R, Sarafov S, Nakov V, Gospodinova M, Todorov T, Kirov A, et al. Transthyretin amyloidosis with gastrointestinal manifestation: A case report. J Gastrointest Liver Dis. 2019;28(3):359-61.

25. Gendre T, Planté-Bordeneuve V. Strategies to improve the quality of life in patients with hereditary transthyretin amyloidosis (hATTR) and autonomic neuropathy. Clin Auton Res. 2019;29:25-31.

26. Adams D, Cauquil C, Labeyrie C. Familial amyloid polyneuropathy. Curr Opin Neurol. 2017;30(5):481-9.

27. Gertz MA, Benson MD, Dyck PJ, Grogan M, Coelho T, Cruz $M$, et al. Diagnosis, Prognosis, and Therapy of Transthyretin Amyloidosis. J Am Coll Cardiol. 2015;66(21):2451-66.

28. Conceição I, González-Duarte A, Obici L, Schmidt HHJ, Simoneau D, Ong ML, et al. Red-flag symptom clusters in transthyretin familial amyloid polyneuropathy. J Peripher Nerv Syst. 2016;21(1):5-9. 
29. Sekijima Y. Transthyretin (ATTR) amyloidosis: Clinical spectrum, molecular pathogenesis and diseasemodifying treatments. J Neurol Neurosurg Psychiatry. 2015;86(9):1036-43.

30. Obici L, Suhr OB. Diagnosis and treatment of gastrointestinal dysfunction in hereditary TTR amyloidosis. Clin Auton Res. 2019;29(0123456789):5563.

31. Plante-Bordeneuve V. Transthyretin familial amyloid polyneuropathy: an update. J Neurol. 2018;265(4):97683.
32. Kerschen P, Planté-Bordeneuve V. Current and Future Treatment Approaches in Transthyretin Familial Amyloid Polyneuropathy. Curr Treat Options Neurol. 2016;18(12).

33. Vollmar J, Schmid JC, Hoppe-Lotichius M, Barreiros AP, Azizi M, Emrich T, et al. Progression of transthyretin (TTR) amyloidosis in donors and recipients after domino liver transplantation-a prospective single-center cohort study. Transpl Int. 2018;31(11):1207-15.

34. Mathew V, Wang AK. Inotersen: New promise for the treatment of hereditary transthyretin amyloidosis. Drug Des Devel Ther. 2019;13:1515-25. 